\title{
Oral administration of fermented wild ginseng ameliorates DSS-induced acute colitis by inhibiting NF- $\kappa B$ signaling and protects intestinal epithelial barrier
}

\author{
Myeong A Seong,"\#, Jong Kyu Woo ${ }^{1, \#}$, Ju-Hee Kang ${ }^{2}$, Yeong Su Jang ${ }^{1}$, Seungho Choi ${ }^{3}$, Young Saeng Jang ${ }^{1}$, Taek Hwan Lee , \\ Kyung Hoon Jung ${ }^{4}$, Dong Kyu Kang ${ }^{4}$, Byung Seok Hurh ${ }^{4}$, Dae Eung Kim ${ }^{4}$, Sun Yeou Kim ${ }^{1}$ E Seung Hyun Oh ${ }^{1, *}$ \\ ${ }^{1}$ Gachon Institute of Pharmaceutical Science, Gachon University, Incheon 406-840, ${ }^{2}$ Research Institute, National Cancer Center, Goyang \\ 410-769, ${ }^{3}$ College of Veterinary Medicine, Seoul National University, Seoul 151-742, ${ }^{4}$ Sempio Fermentation Research center, Osong \\ 363-954, Korea
}

\begin{abstract}
Ginseng has been widely used for therapeutic and preventive purposes for thousands of years. However, orally administered ginseng has very low bioavailability and absorption in the intestine. Therefore, fermented ginseng was developed to enhance the beneficial effects of ginseng in the intestine. In this study, we investigated the molecular mechanisms underlying the anti-inflammatory activity of fermented wild ginseng (FWG). We found that FWG significantly alleviated the severity of colitis in a dextran sodium sulfate (DSS)-induced colitis mouse model, and decreased expression level of pro-inflammatory cytokines in colonic tissue. Moreover, we observed that FWG suppressed the infiltration of macrophages in DSS-induced colitis. FWG also attenuated the transcriptional activity of nuclear factor- $\mathrm{KB}(\mathrm{NF}-\mathrm{\kappa} B)$ by reducing the translocation of NF- $\kappa$ B into the nucleus. Our data indicate that FWG contains anti-inflammatory activity via NF- $\kappa B$ inactivation and could be useful for treating colitis. [BMB Reports 2015; 48(7): 419-425]
\end{abstract}

\section{INTRODUCTION}

Inflammatory bowel disease (IBD) is a chronic inflammatory disease of the gastrointestinal tract such as Crohn's disease and ulcerative colitis (1). The number of patients with IBD is rising in developed countries, and IBD is a public health problem

*Corresponding author. Tel: +82-32-899-6058; Fax: +82-32-8996039; E-mail: eyeball@hanmail.net, eyeball@gachon.ac.kr

${ }^{\#}$ Both authors contributed equally to this work

http://dx.doi.org/10.5483/BMBRep.2015.48.7.039

Received 3 March 2015, Revised 12 March 2015, Accepted 27 April 2015

Keywords: Colitis, Fermented wild ginseng, Inflammation, Macrophage, NF-kB worldwide (2). The existing therapy for IBD often has marginal results, with a high likelihood of additional side effects. Therefore, it is important to develop a new effective and safe therapy for IBD. Recently, herbal medicines/supplements are being used as alternative treatment for IBD (3). Although the cause of IBD is not fully known, several lines of evidence suggest that IBD is due to mucosal barrier dysfunctions, leading to improper immune responses to intestinal microflora, modifications in the composition of microflora, or alterations in tight junction protein expression. These damaged barrier functions are due to high expression of pro-inflammatory cytokines, such as TNF- $\alpha$ and IL-1 $\beta$ (4).

Dextran sodium sulfate (DSS)-induced colitis model is generally a good model of experimental colitis because it has similar symptoms as human IBD (5). Histologically, the DSS-induced colitis model is defined by crypt damage, epithelial ulceration and mucosal edema in association with the infiltration of inflammatory cells such as macrophages $(6,7)$. These processes allow the entry of bacteria into the mucosal layer, which causes the activation of intestinal macrophages $(8,9)$.

Panax ginseng C. A. Meyer (Korea or Asian ginseng) has been widely used as an herbal medicine for centuries in East Asia. The pharmacological effects of ginseng have been confirmed in cancer (10), obesity (11), cardiovascular diseases (12) and inflammation (13). Ginsenosides, metabolites of ginseng, are known as the major active compounds, with a number of pharmacological activities including anti-cancer and anti-inflammatory properties (14). Fermented ginseng has been developed to improve the beneficial effects of ginseng with increased amounts of various ginsenosides such as Rh1, and Compound $\mathrm{K}(\mathrm{C}-\mathrm{K})(15,16)$. Although many comparative studies have been conducted on the therapeutic aspects of ginseng and fermented ginseng, there are no reports on the effects of fermented wild ginseng (FWG) on IBD.

Therefore, we investigated the anti-inflammatory effects of FWG on a DSS-induced colitis mouse model by examining histological changes and inflammatory responses such as the production of pro-inflammatory cytokines and the infiltration of 
macrophages. Furthermore, we used LPS-induced RAW264.7 and mouse peritoneal macrophages to explore the molecular mechanism of FWG in inflammatory conditions.

\section{RESULTS}

\section{Changes in ginsenosides composition from fermented wild ginseng}

Many studies have reported that the conversion of major ginsenosides in ginseng into minor metabolites through the fermentation process improved bioactivity and bioavailability. To investigate whether our fermentation process increases the amount of minor metabolites, we analyzed the minor ginsenoside metabolite contents of wild ginseng (WG) and FWG using HPLC. During fermentation, the contents of C-K, 20(S)-protopanaxatriol (PPT), Rh1, F1 and 20(S)-protopanaxadiol (PPD), which are ginsenosides absent in WG, increased drastically in FWG from $0 \mathrm{mg} / \mathrm{g}$ to $3.32,1.16,0.89,1.5$, and $1.3 \mathrm{mg} / \mathrm{g}$, respectively (Fig. 1A). To investigate whether the increased amount of minor ginsenosides in FWG have a greater suppressive effect on the inflammatory response than WG, we investigated p65 phosphorylation levels in WG and FWG treatment. We found that FWG has a significant inhibitory effect against LPS-induced inflammatory responses in RAW264.7 compared with WG. The results show that FWG contain more active ginsenosides than non-fermented wild ginseng, suggesting that FWG may have more therapeutic effects.

\section{FWG ameliorates DSS-induced colitis in mice}

To evaluate the effect of FWG on DSS-induced colitis, mice were pretreated with FWG (100 mg/kg/day, P.O.) for 3 weeks.
The mice were then exposed to DSS $(2 \%)$ in their drinking water for 7 days with or without FWG. The DSS-treated mice developed acute colitis with severe inflammation, and crypt damage. These changes were reduced by pre-treatment with FWG. The histological score was significantly lower in the FWG-pretreated group $(4.25 \pm 0.89, \mathrm{P}<0.005)$ compared with the DSS-treated group $(7 \pm 0.53)$ (Fig. 1B). These results show that FWG treatment attenuated the severity of DSS-induced colitis.

\section{FWG inhibits pro-inflammatory responses and prevents loss of ZO-1 in colon of DSS-treated mice}

Increase in pro-inflammatory cytokines is a hallmark of inflammation in IBD (17). Therefore, we investigated the effects of FWG on pro-inflammatory cytokine production in the DSS-induced colitis model. Pre-treatment with FWG down-regulated DSS-induced mRNA levels of IL-1 $\beta$, IL-6, and IL-12p40, TNF- $\alpha$ and IFN- $\gamma$, which are pro-inflammatory cytokines in colonic tissues (Fig. 2A). Similarly, we observed that FWG administration led to a decrease in DSS-induced TNF- $\alpha$ protein level in colonic tissue (Fig. 2B). In addition, FWG reduced the number of $\mathrm{F} 4 / 80^{+}$macrophages in DSS-treated mice compared to mice treated with only DSS. This means that FWG suppressed macrophage infiltration into the colon in DSS-treated mice (Fig. 2C). These data suggest that FWG alleviates DSS-induced colitis by controlling innate immunity, such as by regulating macrophage-produced cytokines including TNF- $\alpha$.

Several studies reported that IBD was characterized by a reduction in ZO-1, a tight junctional protein. Alteration of tight junction complexes induces dysfunction in the epithelial bar-

A

\begin{tabular}{ccc}
\hline $\begin{array}{c}\text { Ginsenoside } \\
\text { metabolites }\end{array}$ & $\begin{array}{c}\text { WG } \\
(\mathrm{mg} / \mathrm{g})\end{array}$ & $\begin{array}{c}\text { FWG } \\
(\mathrm{mg} / \mathrm{g})\end{array}$ \\
\hline C-K & N.D & 3.32 \\
PPT & N.D & 1.16 \\
Rh1 & N.D & 0.89 \\
F1 & N.D & 1.5 \\
PPD & N.D & 1.3 \\
\hline
\end{tabular}

B

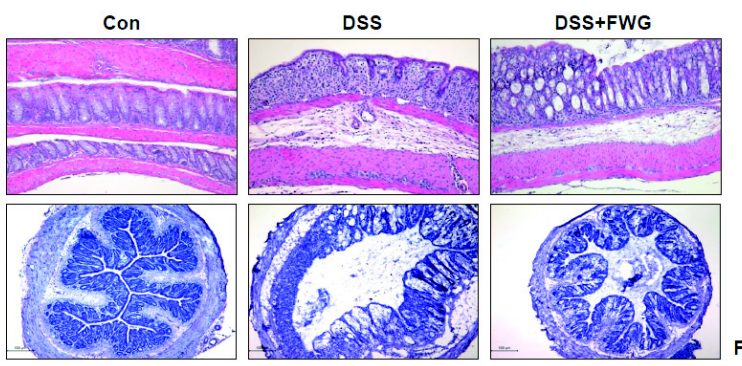

FWG $(100$ (2)

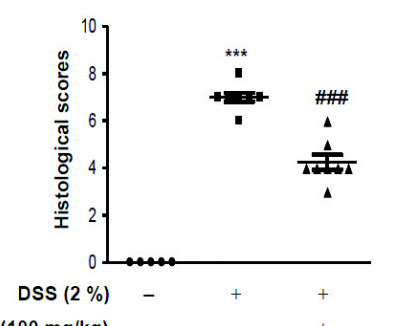

Fig. 1. Fermented wild ginseng (FWG) ameliorates DSS-induced colitis. (A) Minor ginsenoside metabolite contents of wild ginseng (WG) and FWG (B) Mice were administered $2 \%$ DSS in drinking water for 7 days with or without FWG $(100 \mathrm{mg} / \mathrm{kg})$. The paraffin sections were stained with hematoxylin and eosin for histological scores. Longitudinal section (upper, magnification, $\times 200$ ) and cross section (lower, magnification, x100). Values are expressed as mean $\pm \mathrm{SD}$, $\mathrm{n}=8 . * * * \mathrm{P}<0.001$, versus vehicle-treated mice. ${ }^{\# \#} \mathrm{P}<0.001$, versus DSS-treated mice. 


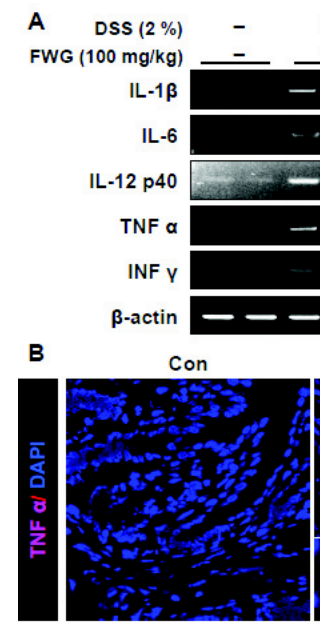

C

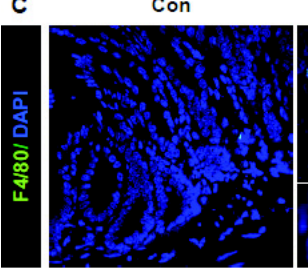

D

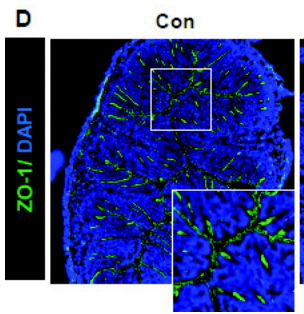

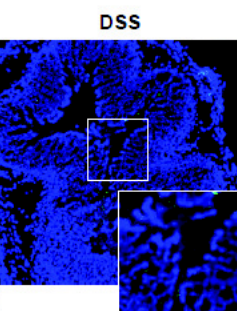

DSS

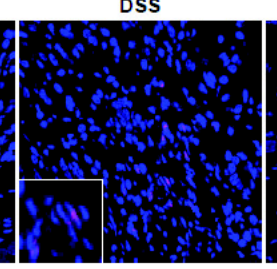

DSS

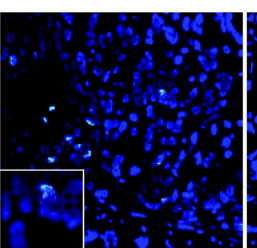

DSS

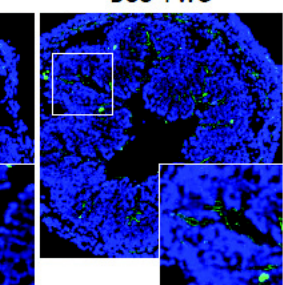

Fig. 2. FWG suppressed production of pro-inflammatory cytokines and recovers lost ZO-1 in colon of DSS-induced colitis mice. (A) RT-PCR analysis was used for the determination of pro-inflammatory cytokine mRNA levels. (B) Immunofluorescence staining of representative frozen sections for the determination of TNF $\alpha$ level (red), (C) F4/80 (green) positive macrophage infiltrations and (D) ZO-1 distribution/expression; control, $2 \%$ DSS treated and 2\% DSS plus $100 \mathrm{mg} / \mathrm{kg}$ FWG treated mice. Nuclei were stained with DAPI (blue); (B, C) Magnification, $\times 200$, (D) Magnification, $\times 40$.

rier $(18,19)$. To further explore the beneficial effects of FWG on the DSS-induced colitis model, we studied whether pre-treatment with FWG had a protective effect on the intestinal barrier in DSS-mediated colitis. We found that FWG pre-treatment prevented DSS-mediated zonula occludens-1 (ZO-1) reduction from tight junction complexes in colonic epithelial cells (Fig. 2D). The results suggest that FWG is involved in the distribution of tight junction complexes and improve intestinal barrier functions.
A

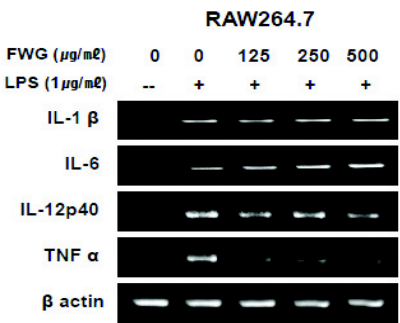

B

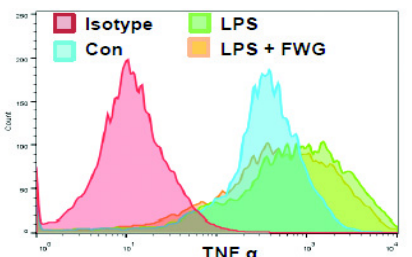

C

Con
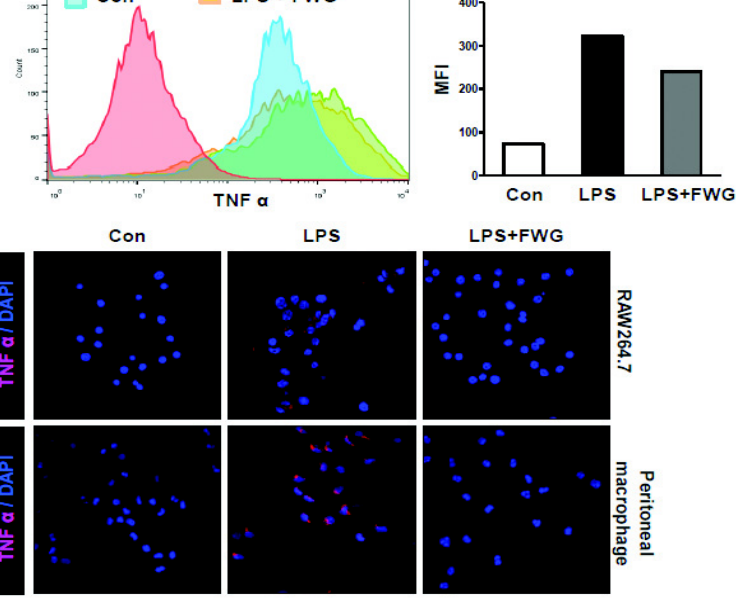

LPS+FWG

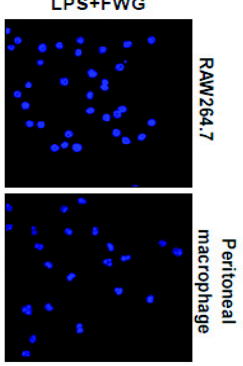

Fig. 3. FWG reduces production of pro-inflammatory cytokines in RAW264.7 macrophages and peritoneal macrophages in vitro. The cells were pretreated with FWG for $1 \mathrm{~h}$ and then induced with LPS $(1 \mu \mathrm{g} / \mathrm{ml})$ for $4 \mathrm{~h}$. (A) mRNA levels of pro-inflammatory cytokines were determined by RT-PCR. (B) TNF- $\alpha$ level in RAW 264.7 cell line was determined by FACS analysis. Histogram shows mean fluorescence intensity (MFI) of TNF- $\alpha^{+}$cells. (C) TNF- $\alpha$ level (red) in RAW264.7 and peritoneal macrophages were determined by immunofluorescence assay. Nuclei were stained with DAPI (blue). Magnification, $\times 600$.

FWG reduces production of pro-inflammatory cytokines in RAW264.7 macrophage and peritoneal macrophage in vitro It was reported that LPS-stimulated production of pro-inflammatory cytokines is caused by NF- $\mathrm{kB}$ activation and suppression of NF- $\mathrm{KB}$ activity leads to reduction of those cytokines (20). To identify the mechanism involved in the anti-inflammatory response by FWG, we investigated whether FWG inhibits LPS-induced pro-inflammatory cytokine production in vitro. FWG decreased LPS-induced up-regulation of TNF- $\alpha$ and IL-12 $\mathrm{p} 40$, but not IL-1 $\beta$ and IL-6, at the mRNA levels in a concentration dependent manner in RAW264.7 macrophages. In peritoneal macrophages, pre-treatment with FWG reduced LPS-induced up-regulation of IL-1 $\beta$, IL-6, IL-12p40, TNF- $\alpha$, and IFN- $\gamma$ mRNA levels (Fig. 3A). Similarly, FWG suppressed LPS-induced up-regulation of TNF- $\alpha$ protein level as shown in FACS and immunofluorescence assays in RAW264.7 macrophages 


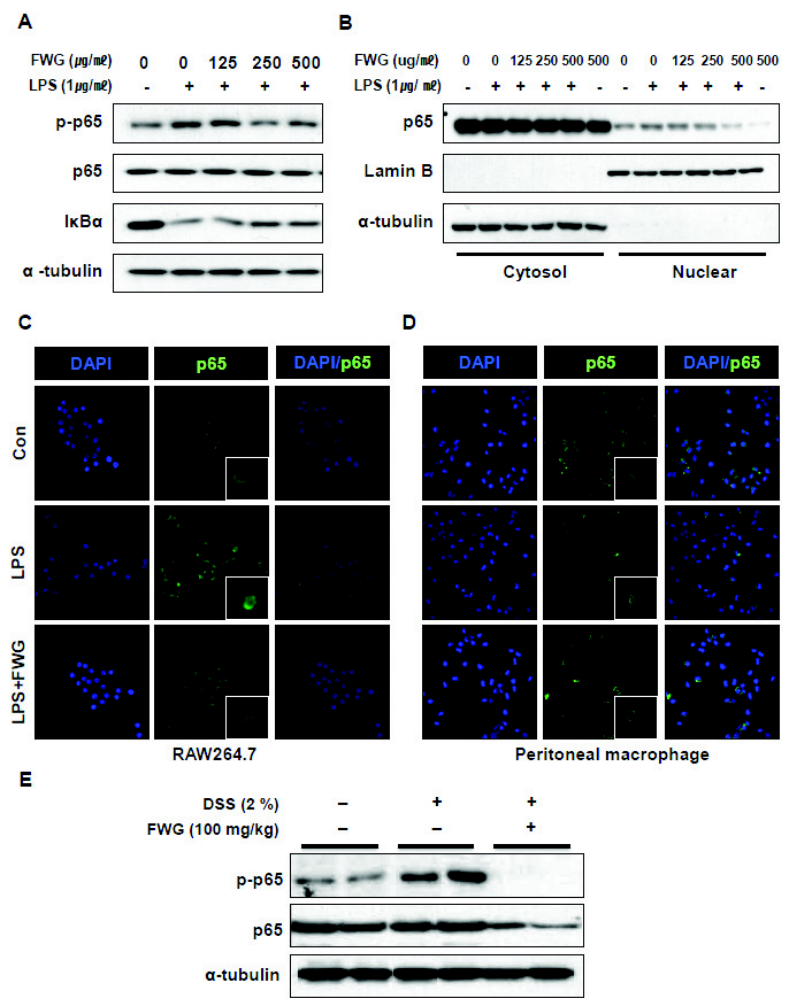

Fig. 4. FWG inhibits the NF- $\kappa B$ activation in inflammatory conditions in vitro and in vivo. (A) RAW264.7 macrophage cell lines were pretreated with FWG $(125,250,500 \mu \mathrm{g} / \mathrm{ml})$ for $1 \mathrm{~h}$ and then stimulated with LPS $(1 \mu \mathrm{g} / \mathrm{ml})$ for $30 \mathrm{~min}$. Western blot analysis for I $1 \kappa-\alpha, p-p 65$, and p65 are shown. (B) RAW264.7 macrophage cell lines were pretreated with FWG $(125,250,500 \mu \mathrm{g} / \mathrm{ml})$ for $1 \mathrm{~h}$ and stimulated with LPS $(1 \mu \mathrm{g} / \mathrm{ml})$ for $1 \mathrm{~h}$. Nuclear and cytosolic extracts were isolated and the levels of p65 in each fraction were determined by western blot. Lamin B and $\alpha$-tubulin were used as internal controls. (C) RAW264.7 macrophage cell line and (D) peritoneal macrophage were pretreated with 250 $\mu \mathrm{g} / \mathrm{ml}$ FWG for $1 \mathrm{~h}$ and then stimulated with LPS $(1 \mu \mathrm{g} / \mathrm{ml})$ for 1 h. NF-kB nuclear translocation was detected by immunofluorescence assay using an antibody for p65 (green). Nuclei were stained with DAPI (blue). Magnification, $\times 600$. (E) Total proteins were prepared from colon tissues of control, $2 \%$ DSS treated and $2 \%$ DSS plus $100 \mathrm{mg} / \mathrm{kg}$ FWG treated mice. The levels of p-p65 and p65 were determined by western blot.

and peritoneal macrophages from mice (Fig. 3B, C). Consistent with the in vivo data, we confirmed that treatment with FWG reduced the expression of pro-inflammatory cytokines in vitro.

FWG suppress NF- $\mathrm{KB}$ signaling pathway in vitro and in vivo The activation of NF- $\kappa B$ is significantly induced in IBD patients (21). Therefore, we investigated whether FWG abrogates $\mathrm{NF}-\mathrm{\kappa B}$ signaling in vitro. We found that pretreatment with FWG inhibited LPS-induced degradation of $I_{\kappa} \mathrm{B}-\alpha$ and phosphorylation of NF-kB (Fig. 4A). We also found that FWG significantly suppressed the LPS-induced translocation of the
NF- $\kappa \mathrm{B}$ subunit, p65, to the nucleus in RAW264.7 (Fig. 4B, C) and peritoneal macrophages (Fig. 4D). We further investigated whether pretreatment with FWG inhibits the activation of NF- $\mathrm{KB}$ signaling in DSS-induced colitis mice. In agreement with our in vitro results, pretreatment with FWG prevented DSS-induced NF- $\kappa B$ phosphorylation in colon tissues (Fig. 4E). These data showed that FWG suppressed the NF- $\mathrm{KB}$ signaling pathway by inhibiting the translocation of NF-KB.

\section{DISCUSSION}

Many studies have reported that various herbal medicines such as aloe vera gel, Boswellia serrata and wheat grass juice seem to be effective in the treatment of IBD (22). However, available evidence regarding herbal medicine is complex and incomplete, therefore, there is a need for further investigation to maximize the quality and efficacy. Ginseng is wildly used for traditional medicines. Ginsenosides, the major components of ginseng, have been reported to have a range of pharmacological and therapeutic effects such as anti-inflammation and anti-cancer activities. Recently, some studies presented evidence that ginseng has therapeutic effects on inflammation associated with IBD (23). However, the bioavailability of ginsenosides in humans after oral administration of ginseng is very low (24). Therefore, many studies have suggested methods such as mild acid hydrolysis as well as enzymatic and microbial conversion to improve the bioactivity and bioavailability of ginsenosides (25). Consequently, we developed fermented wild ginseng through enzymatic conversion (fermentation), which is a highly selective method that utilizes Aspergillus niger, Aspergillus oryzae and Penicillum sp derived enzymes. Based on our HPLC analysis, we found that our fermentation process could convert the major ginsenosides, Re, Rg1, Rg2, $\mathrm{Rc}$, and Rb2 into ginsenoside metabolites, Rh1, F1, C-K, PPT, and PPD which are active forms of the ginsenosides (Fig. 1A). It was recently reported that the conversion of $\mathrm{Rb} 1, \mathrm{Rb} 2$, and Rc into their deglycosylated metabolite C-K could considerable increase its bioactivity and bioavailability (26). In addition, $\operatorname{Rg} 1$ and $\operatorname{Re}$ are metabolized to Rh1 or F1, which are more easily absorbed into the bloodstream and act in target tissues (21). Among ginsenoside metabolites, C-K and Rh1 in particular are more active than other metabolites against inflammation $(25,27)$. There is already a report that C-K suppresses the inflammatory response and has therapeutic effects in DSS-induced colitis (28). This suggests that FWG may be biochemically and functionally superior to raw material, especially against inflammation.

To investigate the anti-inflammatory effects of FWG in IBD, we used a DSS-induced colitis mouse model, which has symptoms similar to human colitis. Our data showed that FWG treatment significantly suppressed DSS-induced colonic inflammation and crypt damage (Fig. 2B). Some studies suggest that activation of intestinal macrophages may be involved in the major pathway of IBD (29). Activated macrophages are 
considered major mediators in the production of pro-inflammatory cytokines such as IL-1 $\beta$, IL-6, IL-12 p40, and TNF- $\alpha$ in the gut. Excessive production of cytokines is the pathogenesis for IBD (30). For this reason, the regulation of inflammatory cytokines production has been largely studied and is even regarded as a new therapeutic target for the treatment of IBD. We observed an increase in the levels of pro-inflammatory cytokines in the colon tissue of DSS-induced mice and FWG treatment reduced the expression of pro-inflammatory cytokines at the mRNA and protein levels (Fig. 2A, B). Furthermore, FWG suppressed DSS-induced macrophage infiltration (Fig. 2C).

IBD is related to damages in the intestinal barrier, which regulates the infiltration of endotoxins or gram-negative bacteria (31). Tight junction complexes support the intestinal epithelial barrier and are formed by transmembrane and intracellular proteins such as ZO-1 $(32,33)$. Therefore, we investigated the distribution and expression of the tight junction protein, ZO-1. As previously reported (19), we observed the loss of ZO-1 expression in DSS-induced mice. Interestingly, treatment with FWG recovered the expression and distribution of ZO-1 in the colon of DSS-induced mice (Fig. 2D), suggesting that the regulation of colitis by FWG might be due to improvements in the function of the intestinal epithelial barrier.

A number of studies have reported that NF- $\mathrm{KB}$ is substantially activated in DSS-induced colitis and in patients with IBD, and the levels of activated NF- $\mathrm{KB}$ are associated with the severity of inflammation (21). The activation of NF- $\mathrm{kB}$ causes the production of inflammatory mediators including the recruitment of pro-inflammatory cytokines and the activation of immune cells such as macrophages. This suggests that blocking NF- $\kappa \mathrm{B}$ signaling might be a potential target for the treatment of IBD (32). We found that FWG exerts inhibitory effects on these inflammatory responses via the suppression of NF- $\mathrm{KB}$ activation in DSS-induced mice and LPS-stimulated RAW264.7 macrophages (Fig. 4), suggesting that the anti-colitic effects of FWG may be related to the inhibition of macrophage activation via NF-кB.

In conclusion, the present study demonstrated the anti-inflammatory activity and regulation of intestinal barrier by FWG in DSS-induced mice. Accordingly, we conclude that FWG can be a useful compound for the prevention and treatment of IBD.

\section{MATERIALS AND METHODS}

\section{Reagents and Materials}

DSS (molecular weight 36-50 kDa) was purchased from MP Biomedical (OH, USA). DMEM, RPMI 1640, fetal bovine serum (FBS), penicillin (100 unit/ml) and streptomycin $(100 \mu \mathrm{g} / \mathrm{ml})$ were obtained from Welgene Inc. (Daegu, Korea). LPS purified from Escherichia coli O111:B4 was purchased from SigmaAldrich (MO, USA). The following antibodies were used in these studies: p-p65 (Cell signaling, MA, USA), ІкB $\alpha$ (Cell signaling, MA, USA), p65 (Santa Cruz, TX, USA), ZO-1 (Invitrogen,
CA, USA) and TNF $\alpha-$ PE (BD Biosciences, CA, USA).

\section{Preparation of fermented wild ginseng (FWG)}

Cultured wild ginseng root, generated from tissue cultures of Korean wild ginseng, was purchased from Vitrosis Co., LTD. (Young-ju, Korea) in Nov 2013. The wild ginseng was completely pulverized (particle size below $1,000 \mu \mathrm{m}$ ). The ginseng powder was incubated with cellulase and hemi-cellulase at $60^{\circ} \mathrm{C}$ for $6 \mathrm{~h}$ and then pretreated in a pressurized condition at $125^{\circ} \mathrm{C}$ for $30 \mathrm{~min}$. The pretreated powder was fermented through the process of enzyme reaction with lactase, beta-glucosidase, and naringinase derived from Aspergillus niger, Aspergillus oryzae and Penicillum sp. The product was pulverized again to particle sizes below $1 \mu \mathrm{m}$. The fermented wild ginseng powder (FWG) was sterilized using an autoclave at $121^{\circ} \mathrm{C}$ for $15 \mathrm{~min}$. For treatment, the FWG was dissolved in DMSO and filtered with a syringe filter (pore size $0.22 \mu \mathrm{m}$ ).

\section{HPLC analysis}

To measure the changes in ginsenoside composition in fermented wild ginseng, HPLC analysis was carried out on an Agilent 1200 series (Agilent, Santa Clara, CA, USA) LC system equipped with a separation module with an integrated column heater, an autosampler, and a photodiode array detector. UV absorbance was monitored at 200 to $400 \mathrm{~nm}$. Quantification was performed by integrating the peak areas at $203 \mathrm{~nm}$. The injection volume was $10 \mu \mathrm{l}$. A column (Thermo Hypersil gold ODS-H80, $150 \times 4.6 \mathrm{~mm}$; particle size, $5 \mathrm{um}$; Thermo Electron Corporation, Cheshire, UK) was installed in a column oven and maintained at $45^{\circ} \mathrm{C}$. The mobile phase was composed of water (solvent A) and acetonitrile (solvent B). The flow rate was $1.0 \mathrm{ml} / \mathrm{J}$. The gradient was $0.0 \mathrm{~min}, 5 \% \mathrm{~B} ; 10$ $\min , 5 \%$ B; $40.0 \mathrm{~min}, 32 \% \mathrm{~B} ; 55.0 \mathrm{~min}, 60 \% \mathrm{~B} ; 70.0 \mathrm{~min}$, $75.0 \%$ B; $72.0 \mathrm{~min}, 90.0 \% \mathrm{~B} ; 82.0 \mathrm{~min}, 90.0 \% \mathrm{~B} ; 84.0 \mathrm{~min}$, $5.0 \% \mathrm{~B}$ and $90.0 \mathrm{~min}, 5 \% \mathrm{~B}$.

\section{Cell culture}

RAW264.7 murine macrophages were obtained from the Korea Cell Line Bank (Seoul, Korea). Cells were grown at $37^{\circ} \mathrm{C}$ in RPMI supplemented with $10 \%$ FBS, penicillin (100 units/ml) and streptomycin $(100 \mu \mathrm{g} / \mathrm{ml})$ in a humidified atmosphere of $5 \% \mathrm{CO}_{2}$.

\section{Experimental animal}

Six-week old female C57BL/6J mice were received from the Orient Co. (Charles River Laboratories). All animal procedures were performed according to the approved protocols [LCDI2013-0045] and institution recommendations for the proper use and care of laboratory animals, Center of Animal Care and Use, College of Pharmacy, Gachon University.

\section{Induction of colitis}

Experimental colitis was induced by exposing mice to drinking water containing $2 \%(\mathrm{w} / \mathrm{v})$ DSS for 7 days. For each experi- 
ment, the mice were divided into 3 groups. The first group was the vehicle-treated control, and the second group was given drinking water with DSS only. The last group consisted of mice pretreated with $100 \mathrm{mg} / \mathrm{kg}$ FWG (5 times a week, P.O.) for 3 weeks, and then $2 \%$ DSS with $100 \mathrm{mg} / \mathrm{kg}$ FWG for 1 week according to the experimental design. The experiments were conducted twice, with 6 mice in each group.

\section{Histological analysis of colon}

Paraffin-embedded tissue sections of swiss-rolled whole colon were stained with hematoxylin and eosin for histological scoring. Samples from the entire colon were examined in a blinded fashion. Colitis assessment was based on criteria including the degree of inflammation (scale of 0-3), edema (0-4) and crypt damage (0-4), as described previously (34).

\section{RNA isolation and RT-PCR}

Total RNA was isolated using TRIzol reagent (Invitrogen, CA, USA), according to the manufacturer's protocol. First strand cDNA was synthesized with the PrimeScript RT reagent Kit (TaKaRa, Shiga, Japan) using $3 \mu$ g total RNA according to the manufacturer's protocol. cDNA was then amplified by PCR with mouse specific primers: IL-1 $\beta$, GCC TTG GGC CTC AAA GGA AAG AAT C (forward) and GGA AGA CAC AGA TTC CAT GGT GAA G (reverse); IL-6, TGG AGT CAC AGA AGG AGT GGC TAA G (forward) and TCT GAC CAC AGT GAG GAA TGT CCA C (reverse); IL-12p40, GTC CTC AGA AGC TAA CCA TC (forward) and TIT CCA GAG CCT ATG ACT CC (reverse); TNF- $\alpha$, ATA GCT CCC AGA AAA GCA AGC (forward) and CAC CCC GAA GTT CAG TAG ACA (reverse); INF $\gamma$ : AGC GGC TGA CTG AAC TCA GAT TGT AG (forward) and GTC ACA GTT TTC AGC TGT ATA GGG (reverse); $\beta$-actin: TGG AAT CCT GTG GCA TCC ATG AAA C (forward) and TAA AAC GCA GCT CAG TAA CAG TCC G (reverse). The RT-PCR exponential phase was determined on cycles 28-33 to allow quantitative comparisons among the cDNAs amplified from identical reactions.

\section{Western blot analysis}

Whole cell, cytoplasmic and nuclear extracts were prepared as described previously (35). Protein extract lysates were resolved by SDS-polyacrylamide gel electrophoresis and transferred to polyvinylidene fluoride membranes. The protein-antibody complexes were detected by Absignal (Abclone, Seoul, Korea) according to the manufacturer's recommended protocol.

Analysis of flow cytometry for intracellular cytokine detection For intracellular cytokine staining, RAW264.7 were resuspended in PBS containing 0.05\% Tween 20 and incubated for 30 min. After washing in PBS, cells were incubated with anti-mouse TNF $\alpha$-PE with $0.02 \%$ Tween 20 solution for 30 min on ice. Cells were subjected to flow cytometry analysis on a FACSCalibur cytometer (BD Biosciences, CA, USA). 10,000 events were acquired and analyzed using the Flowzo data analysis software (Tree Star, OR, USA).

\section{Immunofluoresence assay}

In RAW264.7, cells grown on glass coverslips were fixed and made permeable in methanol at $-20^{\circ} \mathrm{C}$. The coverslips were incubated with anti-p65 antibody overnight at $4^{\circ} \mathrm{C}$. Colon sections were fixed with $4 \%$ para-formaldehyde for $1 \mathrm{~h}$ then stained with primary antibody overnight at $4^{\circ} \mathrm{C}$ followed by incubation in FITC-labeled secondary antibody for $1 \mathrm{~h}$ at room temperature. Sections and coverslips were then mounted with mounting medium containing 4,6-diamidino-2-phenylindole (DAPI) for nuclear counterstaining. Images were observed by fluorescence microscopy. FITC and DAPI images were obtained from the same area.

\section{Statistical analysis}

The results are expressed as mean \pm SD values of triplicate experiments. Each treated group was compared with the control group and statistical significance was determined by Student's $t$ tests. Values with $\mathrm{P}<0.001$ were considered significant.

\section{ACKNOWLEDGEMENTS}

This research was supported by the Sempio fermentation research center-2013, and High Value-added Food Technology Development Program 114006-04, Ministry of Agriculture, Food and Rural Affairs.

\section{REFERENCES}

1. Xavier RJ and Podolsky DK (2007) Unravelling the pathogenesis of inflammatory bowel disease. Nature 448, 427-434

2. Schwanke RC, Marcon R, Meotti FC et al (2013) Oral administration of the flavonoid myricitrin prevents dextran sulfate sodium-induced experimental colitis in mice through modulation of PI3K/Akt signaling pathway. Mol Nutr Food Res 57, 1938-1949

3. Ke F, Yadav PK and Ju LZ (2012) Herbal medicine in the treatment of ulcerative colitis. Saudi J Gastroenterol 18, 3-10

4. Strober W, Fuss I and Mannon P (2007) The fundamental basis of inflammatory bowel disease. J Clin Invest 117, 514-521

5. Melgar S, Karlsson L, Rehnström E et al (2008) Validation of murine dextran sulfate sodium-induced colitis using four therapeutic agents for human inflammatory bowel disease. Int Immunopharmacol 8, 836-844

6. Solomon L, Mansor S, Mallon P et al (2010) The dextran sulphate sodium (DSS) model of colitis: an overview. Comparative Clinical Pathology 19, 235-239

7. Boismenu R and Chen Y (2000) Insights from mouse models of colitis. J Leukoc Biol 67, 267-278

8. Camilleri M, Madsen K, Spiller R, Greenwood-Van Meerveld B and Verne GN (2012) Intestinal barrier function in health and gastrointestinal disease. Neurogastroenterol Motil 24, 503-512 
9. Suzuki T (2013) Regulation of intestinal epithelial permeability by tight junctions. Cell Mol Life Sci 70, 631-659

10. Wong VK, Cheung SS, Li T et al (2010) Asian ginseng extract inhibits in vitro and in vivo growth of mouse lewis lung carcinoma via modulation of ERK-p53 and NF-kappaB signaling. J Cell Biochem 111, 899-910

11. Mollah ML, Kim GS, Moon HK et al (2009) Antiobesity effects of wild ginseng (Panax ginseng C.A. Meyer) mediated by PPAR-gamma, GLUT4 and LPL in ob/ob mice. Phytother Res 23, 220-225

12. Karmazyn M, Moey M and Gan XT (2011) Therapeutic potential of ginseng in the management of cardiovascular disorders. Drugs 71, 1989-2008

13. Lee DC and Lau AS (2011) Effects of Panax ginseng on tumor necrosis factor-alpha-mediated inflammation: a mini-review. Molecules 16, 2802-2816

14. Lu JM, Yao Q and Chen C (2009) Ginseng compounds: an update on their molecular mechanisms and medical applications. Curr Vasc Pharmacol 7, 293-302

15. Fu Y, Yin Z, Wu L and Yin C (2014) Fermentation of ginseng extracts by Penicillium simplicissimum GS33 and anti-ovarian cancer activity of fermented products. World J Microbiol Biotechnol 30, 1019-1025

16. Jung HJ, Choi H, Lim HW et al (2012) Enhancement of anti-inflammatory and antinociceptive actions of red ginseng extract by fermentation. J Pharm Pharmacol 64, 756-762

17. Roberts-Thomson IC, Fon J, Uylaki W, Cummins AG and Barry S (2011) Cells, cytokines and inflammatory bowel disease: a clinical perspective. Expert Rev Gastroenterol Hepatol 5, 703-716

18. Schulzke JD, Ploeger S, Amasheh M et al (2009) Epithelial tight junctions in intestinal inflammation. Ann N Y Acad Sci 1165, 294-300

19. Poritz LS, Garver KI, Green C, Fitzpatrick L, Ruggiero F and Koltun WA (2007) Loss of the Tight Junction Protein ZO-1 in Dextran Sulfate Sodium Induced Colitis. J Surg Res 140, 12-19

20. Kwon DJ, Bae YS, Ju SM, Youn GS, Choi SY, Park J (2014) Salicortin suppresses lipopolysaccharide-stimulated inflammatory responses via blockade of NF-kB and JNK activation in RAW 264.7 macrophages. BMB Rep 47, 318-323

21. Atreya I, Atreya R and Neurath MF (2008) NF-kappaB in inflammatory bowel disease. J Intern Med 263, 591-596

22. Ke F, Yadav PK and Ju LZ (2012) Herbal Medicine in the Treatment of Ulcerative Colitis. Saudi J Gastroenterol 18, 3-10

23. Jin $Y$, Kotakadi VS, Ying $L$ et al (2008) American ginseng suppresses inflammation and DNA damage associated with mouse colitis. Carcinogenesis 29, 2351-2359

24. Hering NA, Fromm M and Schulzke JD (2012) Determinants of colonic barrier function in inflammatory bowel disease and potential therapeutics. J Physiol 590, 1035-1044

25. Joh EH, Lee IA, Jung IH and Kim DH (2011) Ginsenoside Rb1 and its metabolite compound $\mathrm{K}$ inhibit IRAK-1 activation-the key step of inflammation. Biochem Pharmacol 82, 278-286

26. Yang Hsu B, Hui Chen C, Jang Lu T and Sun Hwang L (2013) Bioconversion of ginsenosides in the american ginseng (xi yang shen) extraction residue by fermentation with lingzhi (ling zhi, ganoderma lucidum). J Tradit Complement Med 3, 95-101

27. Park EK, Choo MK, Han MJ and Kim DH (2004) Ginsenoside Rh1 possesses antiallergic and anti-inflammatory activities. Int Arch Allergy Immunol 133, 113-120

28. Li J, Zhong W, Wang W et al (2014) Ginsenoside Metabolite Compound K Promotes Recovery of Dextran Sulfate Sodium-Induced Colitis and Inhibits Inflammatory Responses by Suppressing NF-kB Activation. PLoS One 9, e87810

29. Bain CC and Mowat AM (2011) Intestinal macrophages specialised adaptation to a unique environment. Eur J Immunol 41, 2494-2498

30. Mahida YR (2000) The key role of macrophages in the immunopathogenesis of inflammatory bowel disease. Inflamm Bowel Dis 6, 21-33

31. Bischoff SC, Barbara G, Buurman W et al (2014) Intestinal permeability-a new target for disease prevention and therapy. BMC Gastroenterol 14, 189

32. Dijkstra G, Moshage $\mathrm{H}$ and Jansen PL (2002) Blockade of NF-kappaB activation and donation of nitric oxide: new treatment options in inflammatory bowel disease? Scand J Gastroenterol Suppl, 37-41

33. Nam ST, Hwang JH, Kim DH et al (2014) Role of NADH: quinone oxidoreductase-1 in the tight junctions of colonic epithelial cells. BMB Rep 47, 494-499

34. Mishra SK, Kang JH, Kim DK, Oh SH and Kim MK (2012) Orally administered aqueous extract of Inonotus obliquus ameliorates acute inflammation in dextran sulfate sodium (DSS)-induced colitis in mice. J Ethnopharmacol 143, 524-532

35. Lee SJ, Shin JS, Choi HE et al (2014) Chloroform fraction of Solanum tuberosum L. CV Jayoung epidermis suppresses LPS-induced inflammatory responses in macrophages and DSS-induced colitis in mice. Food Chem Toxicol 63, 53-61 